\title{
A Summary of the Main Contents and Existing Problems in the Study of Multi-Energy Coupling in Energy Internet
}

\author{
Lihua $\mathrm{Li}^{1}$, Yuan $\mathrm{Li}^{2}$, Xiu Yang2, Haini Qu${ }^{1}$, Pengfei Zhang1 \\ ${ }^{1}$ State Grid Electric Power Research Institute, SMEPC, Shanghai, China \\ ${ }^{2}$ Shanghai University of Electric Power, School of Electrical Engineering, Shanghai, China \\ Email: 1i_yuan_0911@163.com
}

How to cite this paper: Li, L.H., Li, Y. Yang, X., Qu, H.N. and Zhang, P.F. (2017) A Summary of the Main Contents and Existing Problems in the Study of Multi-Energy Coupling in Energy Internet. Energy and Power Engineering, 9, 693-702. https://doi.org/10.4236/epe.2017.94B075

Received: March 9, 2017

Accepted: March 30, 2017

Published: April 6, 2017

\begin{abstract}
Multi-energy flow (MEF) coupling is one of the key features of the energy Internet and integrated energy systems that are different from smart grids. With the increasing coupling of heterogeneous energy flow, the system characteristics of coupling are becoming more and more obvious and more complicated. The modeling, analysis and control methods of traditional single flow systems have not been applied directly. Therefore, it is necessary to study the modeling of multi-energy flow coupling, the power flow analysis, optimization and control method of heterogeneous energy flow, which plays the role of multi-energy flow synergy to avoid the adverse effects of coupling. This paper summarizes the current research situation of energy Internet at home and abroad from the aspects of modeling of multi-energy flow, power flow calculation and optimal dispatching, and analyzes the existing problems in the research of these aspects.
\end{abstract}

\section{Keywords}

Energy Internet, Integrated Energy System, Multi-Energy Flow, Energy Hub

\section{Introduction}

In the traditional energy system, all kinds of energy coupling is not tight, different energy systems are relatively independent, such as power grid, heat network, natural gas network are different companies to manage and operate, resulting in the overall efficiency of energy use is not high. With the increasing energy and environment problems, in order to improve the overall energy efficiency and renewable energy consumption capacity, the integration in multiple types of energy interconnection and complementary needs increasingly urgent. The de- 
velopment in reality of a variety of energy conversion equipment (such as CHP / CCHP, heat pump, electric heating, electric hydrogen, etc. ) provides a means for a variety of energy interconnection, a wave of energy Internet research also set off in recent years at home and abroad. Energy Internet aims to reduce the economic development of traditional fossil energy dependence, to maximize the efficiency of the use of renewable energy, fundamentally change the current energy production and consumption patterns.

The concept of energy Internet once put forward, domestic and foreign industries and fields have carried out a useful exploration and research. According to the literature [1], the typical cognitive methods of energy Internet are Energy Internet, Internet of Energy, Intenergy and Multi-Energy Internet. Which are separately represented by the following aspects: the energy network structure which is typically represented by the United States FREEDM; the energy Internet information Internet which is typically represented by the European e-energy; the Internet technology and energy network in-depth integration which is typically represented by the Japan's power router; as well as the electrical, thermal, chemical energy combined delivery and optimization which is typically represented by the United Kingdom, Switzerland and other countries of the energy development direction.

\section{The Current Situation of Energy Internet Research}

\subsection{The Current Research in the United States}

In the United States, the research is focused on energy routers and smart grids. It uses power electronics and information technology in the future distribution network level to achieve energy Internet, put forward the concept of energy routers and prototype implementation, through the power electronics technology to achieve the control of the transformer, and use the communication technology to achieve the interaction between routers. In addition, many scholars' Energy Internet research focused on the architecture (prototype) design of energy Internet. Researchers based on Alex Q Huang have developed the FREEDM system, which enables plug-and-play functionality of distributed devices and is intended to be used as a prototype for next generation of the power systemsenergy Internet. The research team at Berkeley has designed an "information network-centric energy network" architecture [2], it uses a distributed control strategy which is different from centralized control to achieve a flexible response to electricity or electricity price signals. The energy company Stem has developed a smart battery that combines high-density lithium-ion batteries with power electronics devices. When it is placed in buildings, it would switch on the base of electricity prices between charge and discharge conditions which reduce the cost of electricity expense.

\subsection{The Current Research in Germany}

Germany is focused on the research of information and communication networks, through information and communication technology to achieve the self- 
regulation of intelligent energy systems, expect to achieve the objectives of integrated digital Internet, computer control and monitoring in the whole process of the energy supply system. It uses the information and communication network to collect information, and analyzes the decision-making to guide the operation of the energy network scheduling. The "E-Energy" technology innovation promotion program which is launched by the German Federal Ministry of Economics and Technology in 2008 is the most representative. Researchers at the Swiss Federal Institute of Technology have designed an information center and energy monitoring center called "Energy Hub" [3] [4] to enable energy compensation, energy buffering, energy conversion, energy control and energy storage for distribution networks. Part of the energy of its input port come from the electricity of distribution network, and the other part of it come from the different forms of energy (electricity, heat) in the distributed energy. The part of energy in output port supply to a variety of different forms of users (electric, hot, cold), another part of it is fed back to the distribution network in the form of electrical energy.

\subsection{The Current Research in Other Countries}

Some other countries have also studied energy Internet, as described in [5], detailing the function of energy router in the aspects of power generation, transmission, substation, distribution, use and so on. According to the location of the energy router installed in the power grid (end user side and power side), the function of the energy router is discussed from the two aspects, and the typical scenario is described. In the inspiration of people's accustomed to the energy Internet and the Internet, literature [6] proposed the "energy package" concept; the author further designed a circuit to achieve the "energy package" transmission, and carried out a simulation experiment to verify its feasibility.

In the country, the relevant departments proposed to build a comprehensive high-voltage power grid as the backbone grid, the strong smart grid which's voltage level grid coordinated development and also has the features of information, automation and interaction, its essence is "UHV + smart grid + clean energy". In order to meet this requirement, the research on energy Internet is mainly for integrated energy system (IES), electricity-heat coupling system, electricity-gas coupling system and electricity-gas-heat coupling system. The main research contents are: energy router, energy hub modeling and computation, the flow calculation of multi-energy flow, the optimal energy flow calculation of multi-energy flow, and the optimal scheduling and coordination planning in the consideration of multi-time scale.

\section{Multi-Energy Flow Coupling}

Energy Internet consists of four tightly coupled complex network systems, namely, power systems, transport systems, natural gas networks and information networks. (Figure 1) In the fields of the traditional electricity, heat, cold, gas and others, the system modeling method is relatively mature. Power grid is mainly represented 


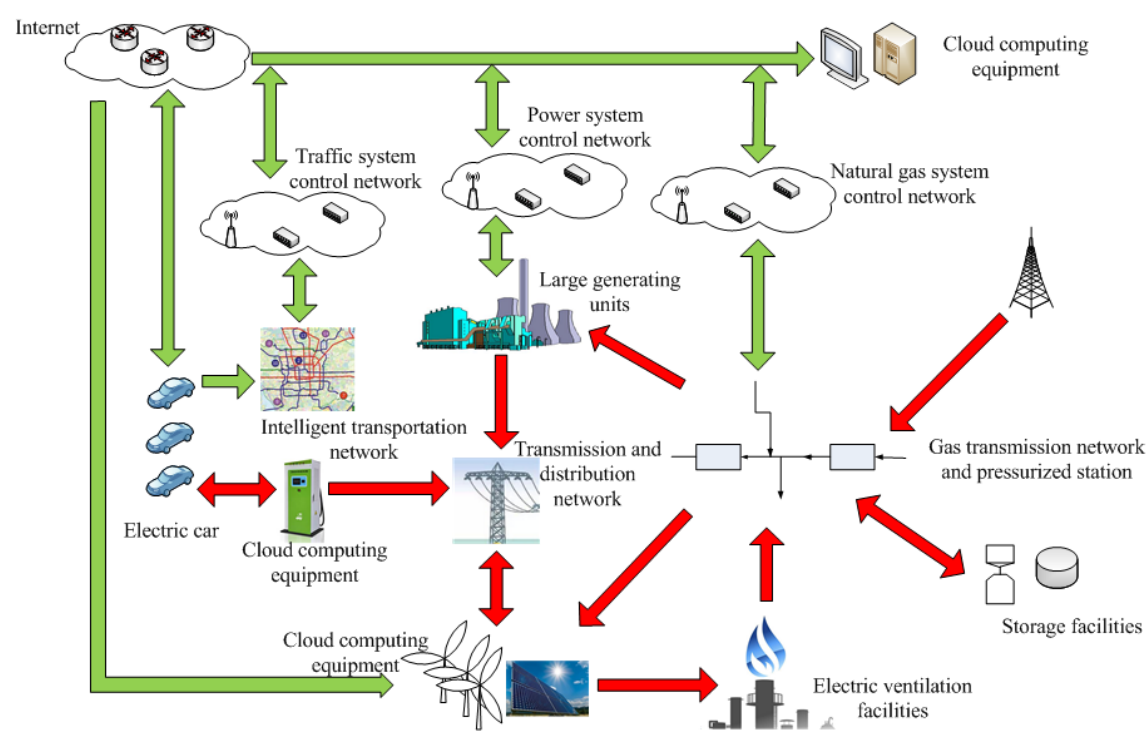

Figure 1. A basic architecture of energy internet.

by the power flow model, follow the law of electromagnetics, the main variables are the voltage amplitude and phase angle of each node, the line active/reactive power flow, the node injection power, etc. It has a set of network analysis methods. The heating/cooling network follows the laws of fluid mechanics and thermodynamics, including the two models of hydraulic and heat, the main variables are pressure, flow, temperature and so on. Natural gas network follows the law of fluid mechanics, the main variables are pressure, flow and so on. The following will analysis the research status about the multi-energy system modeling, power flow calculation and optimization which is related to energy Internet.

\subsection{Modeling}

In recent years, the study of joint modeling and calculation considering the specific characteristic constraints in multi-flow network began to appear. (Figure 2) The power-natural gas (electricity/gas) network as the main energy network in the integrated energy system, the interaction between it has long been concerned by the academic community, in which the literature [7] proposed a natural gas-power hybrid system dynamics model, and studied the interaction between power network and natural gas network in their transient process. In the literature [8], from the point of view of energy supply reliability, the transmission delay and compressibility of natural gas are considered, and the short time optimization operation of the electric/gas coupling system is studied. Although there has been some literature study on the coordinated operation of the electric-gas coupling system, the modeling approach lacks scalability.

In the study of multi-energy systems, the concept of micro-network, virtual power plant (VPP), energy service supply system, intelligent energy network and so on analyze multi-energy system in the overall perspective. Among them, the Swiss Federal Institute of Technology G. Andress on experimental group proposed the concept of energy hub on the multi-energy network description is 


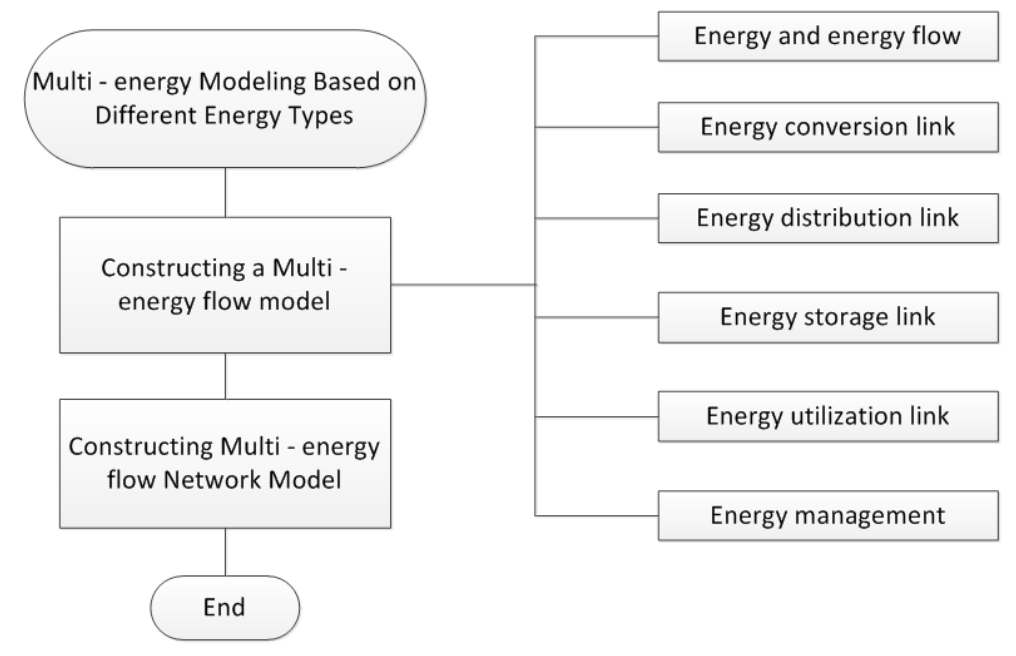

Figure 2. Key link in modeling method based on multi-energy flow model.

more successful, it can be defined as a unit in which different energy can be converted, regulated, stored, it characterizes the interface between different energy facilities and loads, and the coupling between multiple energy systems is represented by a matrix. Literature [9] based on the energy hub concept studied the modeling and analysis of multi-energy system. For the coupling of the aspects "source" and "load", literature [10] [11] proposed a energy center (energy hub) modeling method which can model different energy system. Considering the large time scale difference between electricity, heat and gas in multi-energy flow system, the literature [12] considers the time scale and network constraints, establishes the layered energy management model of regional integrated energy system, but the consideration of the thermal section is limited to the adjustable heat load. In addition, the quasi-steady-state multi-energy model is proposed by taking the time scale characteristic further[13], based on the bus model a modeling method of multi-energy system for general micro-grid is proposed.

\subsection{Energy Flow Calculation}

Previously, there has some preliminary research about the regional integrated energy system analysis and calculation, the corresponding research can be divided into the two categories of unified solution [14] and sequential solution [15]. Among them, the steady-state mixed power flow model of the electric/ thermal coupling system is established in [14]. It studied the joint steady-state multi-energy flow calculation of the power grid and heating network, and proposed two kinds of mixed flow calculation method: unified calculation and sequential calculation. When the unified solution method is adopted, it is necessary to establish a hybrid model of the electric power-gas system. Then, the system flow equation is established under the unified framework, and the comprehensive flow of the system is solved, which is often required in solving the algorithm. When the sequential solution method is used, the power flow equation can be solved separately from the natural gas system. Therefore, the natural gas power flow module and the electricity/gas/heat coupling analysis module can be 
added to the power flow program of the existing power system. In order to fully consider the internal coupling of multi-energy flow, the literature [16] [17] proposed a decomposition algorithm on the optimal energy flow of the electricity /heat/gas mixture with the energy hub as the core, but the method cannot guarantee the global optimal solution. Literature [18] established the energy hub model, respectively, using centralized optimization algorithm and distributed optimization algorithm to solve the optimal power flow of electric/gas hybrid model. Based on the energy hub model, the literature [19] studied the optimal power flow of the integrated network of electricity, natural gas and central heating in the energy Internet. In [20], an electric-thermal-gas mixed power flow algorithm which treat the heat load as a fixed value is proposed, considering the constraints of power network and gas network is in different coupling forms and energy supply modes, the literature gives three modes of operation of the regional integrated energy system: the complete decoupling, partial coupling and full coupling. In addition, considering the large difference of time scale between the three energy sources of electricity, heat and gas in the multi-energy flow system, the literature [21] studied the mechanism and process of influence on the electrical and thermal coupling networks considering the time scale characteristics when various disturbances occurred.

\subsection{Scheduling and Dispatch}

At present, there are many researches on multi-energy flow optimization scheduling at home and abroad, including optimization scheduling model, algorithm and benefit analysis and so on. Under the time-of-use tariff mechanism, the optimization problem of electricity, heat, cold and gas in the family and the change of its peak load are studied [22]. On this basis, the literature [23] takes into account the difference of time scales between electricity and heat, studied the influence of the optimization period. In addition, the comprehensive consideration on a variety of energy to the regional multi-energy system optimization research is conducive to improving the regional energy system economy, reliability, pollution emissions and other indicators, it can also increase the optimize space of system. The literature [24] sets out the control strategy for regional multi-energy system, and the temperature, pressure, flow and other parameters of thermal network is optimized in order to reduce the cost of the system. In [25], the multi-energy system with uncertainty is optimized by using the twolayer optimization strategy of genetic algorithm and Monte Carlo method. In [26], an energy regional optimal scheduling method based on stochastic model predictive control is proposed. In [27], the optimization model of integrated energy system is established, which is based on the two optimal targets of the best economy and environmental protection.

Aiming at the multi-time scale characteristics between electricity, heat and gas energy, the "three-dimensional" optimization scheduling goal considering of time scale, spatial scale and running state is designed in [28], it proposed two optimization scheduling strategy: the spatial dimension optimization scheduling 
strategy of distribution network-feeder-autonomous area and the time dimension optimization scheduling strategy of long time-short time-real-time, and also studied the evaluation method of it. In the literature [29], the multi-time scale distributed power coordination control frame of the active distribution network under normal state is studied, including the global optimal control of active distribution network under long time scale and the regional autonomy control of active distribution under short time scale.

\section{Current Problems}

\subsection{In the Aspect of Modeling}

In the operational phase of natural gas-power hybrid system dynamic model, the $\mathrm{N}-1$ on-line safety analysis for multi-energy flow is still very few, and it cannot meet the need of real-time change of multi-energy flow mode. The energy hub concept facilitates multi-energy network analysis, but it ignores the energy loss in a multi-energy system, which only considering the steady-state analysis. At the same time, from the grid point of view, the traditional energy hub model mainly reflects the active power interaction between energy transmission network and integrated energy system, often does not consider the process of reactive power changes.

\subsection{In the Aspect of Power Flow Calculation}

The mixed power flow algorithm of electricity, heat and gas takes the relevant constraints of power network and gas network in different coupling forms and energy supply modes into account. Although the mixed power flow algorithm is explored, the computational complexity is large, the number of iterations is more and the convergence is difficult to guarantee when the coupling is tight. In addition, most of the steady-state multi-energy flow models are linearized and can be applied to many aspects of planning, evaluation, preliminary optimization, and especially in large-scale systems and strong uncertainties, but these models don't considering the specific characteristics of the different network constraints, the aforementioned electromagnetic, hydrodynamic, thermodynamic constraints are not reflected, so it is difficult to apply to the high accuracy of online energy management, such as the need of solving the multi-energy flow safety analysis.

As a result of the differences of characteristics and the enhancement of coupling in energy flow, how to efficiently solve the multi-energy flow is still a challenge, and there is no mature tool can be used to solve and simulate. The research on the multi-energy flow optimal scheduling at home and abroad has taken the network constraints into account. Some parts of them use the dynamic difference of different energy flow, but it mainly focuses on two kinds of energy coupling and use sea consistent optimization period. The optimization method and the traditional method are more consistent, and the characteristics of multitime scale and multi-management in multi-energy flow is not fully reflected. 


\subsection{In the Aspect of Scheduling and Dispatch}

Some articles have proposed a real-time energy optimization scheduling method. Although real-time scheduling has an unparalleled advantages in terms of efficiency and execution speed, it makes it difficult to avoid the short-sight effect in the scheduling process and also reduce the economy of the system. In the optimal scheduling model, the open-loop scheduling model is adopted. In which the scheduling and optimization of the renewable energy output, the load demand and the price level are regarded as known constants. Due to the influence of the prediction error, it is likely that the proposed optimal scheduling scheme deviates from the actual situation, and the performance of the optimized scheduling model will produce large degradation. Although the open-loop scheduling method of hybrid energy system is proposed in the following, the output of the optimized scheduling model will gradually degrade with the increase of the high permeability of renewable energy due to the difficulty in renewable energy output accurate predictability.

\section{Conclusion}

Multi-energy flow (MEF) is one of the key features of the energy Internet and integrated energy systems that are different from smart grids, which is an important development direction in energy field. The most basic characteristics of multi-energy flow system is that it composed of a variety of heterogeneous energy flow system, and different energy flow systems are coupled together. Heterogeneous energy flow has different models, the difference between characteristics is large, so each of them have different modeling, analysis and control methods. Heterogeneous energy flow convert and act on each other, which coupled to form a more complex large system. The modeling, analysis and control methods of traditional single-stream systems have not been applied directly. Therefore, it is necessary to study the modeling of multi-energy flow coupling, the flow analysis, optimization and control method of heterogeneous energy flow, and play the role of multi-energy flow synergy to avoid the adverse effects of coupling. But this area is still in the initial stage of the study at home and abroad, it is still lacking in systematic research and mature system applications, which has a large research potential. It is also necessary to carry out more comprehensive research on the modeling, flow calculation and optimal scheduling of multi-energy flow coupling, and then to obtain verification and application in the digital simulation platform and actual multi-energy flow system, it is conducive to promoting the use of renewable energy and the comprehensive energy efficiency. It is also conducive to the integrated energy security, it promotes the upgrading of the energy industry, and provides a new technical approach for China's energy production and consumption revolution.

\section{References}

[1] Ma, Z., Zhou, X.X., Shang, Y.W. and Sheng, W.X. (2015) Exploring the Concept, Key Technologies and Development Model of Energy Internet. Power System 
Technology, 3014-3022.

[2] Katz, R.H., Culler, D.E., Sanders, S., et al. An Information-Centric Energy Infrastructure: the Berkeley View. (2011) Sustainable Computing: Informatics and Systems, 1, 7-22.

[3] Geidl, M., Koeppel, G. and Favre-Perrod, P., et al. (2007) Energy Hubs for the Future. IEEE Power and Energy Magazine, 5, 24-30. https://doi.org/10.1109/MPAE.2007.264850

[4] Ghasemi, A., Hojja, T.M. and Javidi, M.H. (2012) Introducing a New Framework for Management of Future Distribution Networks Using Potentials of Energy Hubs. Smart Grids (ICSG), 2012 2nd Iranian Conference on. IEEE, 1-7.

[5] Xu, Y., Zhang, J., Wang, W., et al. (2011) Energy Router: Architectures and Functionalities toward Energy Internet. Smart Grid Communications (SmartGridComm), 2011 IEEE International Conference on. IEEE, 31-36.

https://doi.org/10.1109/SmartGridComm.2011.6102340

[6] Corzine, K.A. (2014) Energy Packets Enabling the Energy Internet. Power Systems Conference (PSC), 2014 Clemson University. IEEE, 1-5.

https://doi.org/10.1109/PSC.2014.6808106

[7] Xu, X., Jia, H. and Chiang, H.D., et al. (2015) Dynamic modeling and interaction of Hybrid Natural Gas and Electricity Supply System In Microgrid. IEEE Transactions on Power Systems, 30, 1212-1221. https://doi.org/10.1109/TPWRS.2014.2343021

[8] Correa-Posada, C.M. and Sanchez-Martin, P. (2015) Integrated Power and Natural Gas Model for Energy Adequacy in Short-Term Operation. IEEE Transactions on Power Systems, 30, 3347-3355. https://doi.org/10.1109/TPWRS.2014.2372013

[9] Krause, T., Andersson, G., Frohlich, K., et al. Multiple-energy Carriers: Modeling of Production, Delivery, and Consumption. Proceedings of the IEEE, 99, 15-27.

[10] Huang, G.R., Liu, W.J., Wen, F.S., et al. (2016) Collaborative Planning of Integrated Electricity and Natural Gas Energy Systems with Power-to-Gas Stations. Electric Power Construction, 9, 1-13.https://doi.org/10.1109/jproc.2010.2083610

[11] Zhang, H., Wen, F.S., Zhang, C., et al. (2016) Operation Strategy for Residential Quarter Energy Hub Considering Energy Demands Uncertainties. Electric Power Construction, 9, 14-21.

[12] Xu, X., Jin, X., Jia, H., et al. (2015) Hierarchical Management for Integrated Community Energy Systems. Applied Energy, 160, 231-243.

https://doi.org/10.1016/j.apenergy.2015.08.134

[13] Wang, C.S., Hong, B.W., Guo, L.et al. (2013) A General Modeling Method for Optimal Dispatch of Combined Cooling, Heating and Power Microgrid. Proceedings of the CSEE, 33, 26-33.

[14] Liu, X., Wu, J., Jenkins, N., et al. (2016) Combined Analysis of Electricity and Heat Networks. Applied Energy, 162, 1238-1250. https://doi.org/10.1016/j.apenergy.2015.01.102

[15] Zhangm, Y.B. (2005) Natural Gas - Power Hybrid System Analysis Method. China Electric Power Research Institute, Beijing.

[16] Moeini-Aghtaie, M., Abbaspour, A., Fotuhi-Firuzabad, M., et al. (2014) A Decomposed Solution to Multiple-Energy Carriers Optimal Power Flow. IEEE Transactions on Power Systems, 29, 707-716.https://doi.org/10.1109/TPWRS.2013.2283259

[17] Shabanpour-Haghighi, A. and Seifi, A.R. (2015) Simultaneous Integrated Optimal Energy Flow of Electricity, Gas, and Heat. Energy Conversion and Management, 101, 579-591. https://doi.org/10.1016/j.enconman.2015.06.002 
[18] Geidl, M. (2007) Integrated Modeling and Optimization of Multi-Carrier Energy Systems. TU Graz.

[19] Sun, Q.Y., Zhao, M.Y., Chen, Y. and Ma, D.Z. Optimal Energy Flow of Multiple Energy Systems in Energy Internet. Proceedings of the CSEE, 1-10.

[20] Xu, X.D., Jia, H.J., Jin, X.L., et al. (2015) Study on Hybrid Heat-Gas-Power Flow Algorithm for Integrated Community Energy System. Proceedings of the CSEE, 35, 3634-3642.

[21] Pan, Z, Guo, Q. and Sun, H. (2016) Interactions of District Electricity and Heating Systems Considering Time-Scale Characteristics Based on Quasi-Steady Multi-Energy Flow. Applied Energy, 167, 230-243. https://doi.org/10.1016/j.apenergy.2015.10.095

[22] Pan, Z., Sun H, Guo Q. Tou-based optimal energy management for smart home[C]//Innovative Smart Grid Technologies Europe (ISGT EUROPE), 2013 4th IEEE/PES. IEEE, 2013: 1-5.

[23] Pan, Z., Guo, Q. and Sun, H. (2015) Impacts of Optimization Interval on Home Energy Scheduling for Thermostatically Controlled Appliances. CSEE Journal of Power and Energy Systems, 1, 90-100. https://doi.org/10.17775/CSEEJPES.2015.00024

[24] Pirouti, M., Bagdanavicius, A., Ekanayake, J., et al. (2013) Energy Consumption and Economic Analyses of A District Heating Network. Energy, 57, 149-159. https://doi.org/10.1016/j.energy.2013.01.065

[25] Zhou, Z., Zhang, J., Liu, P., et al. (2013) A Two-Stage Stochastic Programming Model for the Optimal Design of Distributed Energy Systems. Applied Energy, 103,135-144. https://doi.org/10.1016/j.apenergy.2012.09.019

[26] Zhang, Y., Zhang, T., Liu, Y.J., et al. (2016) Stochastic Model Predictive Control for Energy Management Optimization of an Energy Local Network. Proceedings of the CSEE, 36, 3451-3462.

[27] Yu, B., Wu, L., Lu, X., et al. (2016) Optimal Dispatching Method of Integrated Community Energy System. Electric Power Construction, 1, 70-76.

[28] Pu, T.J.,Chen, N.S., Wang, X.H., et al. (2016) Application and Architecture of Multi-source Coordinated Optimal Dispatch for Active Distribution Network. Automation of Electric Power Systems, 1, 003.

[29] You, Y., Liu, D., Zhong, Q., et al. (2014) Multi-time Scale Cordinated Control of Distributed Generators Based on Active Distribution Network. Automation of Electric Power Systems, 38, 192-198. 
Submit or recommend next manuscript to SCIRP and we will provide best service for you:

Accepting pre-submission inquiries through Email, Facebook, LinkedIn, Twitter, etc. A wide selection of journals (inclusive of 9 subjects, more than 200 journals)

Providing 24-hour high-quality service

User-friendly online submission system

Fair and swift peer-review system

Efficient typesetting and proofreading procedure

Display of the result of downloads and visits, as well as the number of cited articles Maximum dissemination of your research work

Submit your manuscript at: http://papersubmission.scirp.org/

Or contact epe@scirp.org 